\title{
Erratum
}

\section{Ship funds as a new asset class: An empirical analysis of the relationship between spot and forward prices in freight markets}

\section{Wolfgang Bessler Wolfgang Drobetz Jorg Seidel}

Journal of Asset Management (2008) 9, 254. doi:10.1057/jam.2008.21

Correction to: Journal of Asset Management (2008) 9, 102-120, doi:10.1057/jam.2008.14

The editors and publishers would like to apologise to the authors for errors that occurred in the article.

On page 104, second line the word 'kommanaitesellschaft' should read:

'Kommanditgesellschaft'.

On page 106, second column the sentence:

'Therefore, because $S$ cannot surprise $F x$, the spot rate converges to the forward rate...?

should read:

'Therefore, because $S$ cannot surprise $F$, the spot rate converges to the forward rate....'

On page 108, equations 4 and 5:

$$
\begin{aligned}
\Delta S_{t}= & c_{10}+\sum_{i=1}^{k} a_{1 i} \Delta S_{t-i}+\sum_{j=1}^{k} b_{i j} \Delta F_{t-j} \\
& +\gamma_{1} Z_{t-1}+\varepsilon_{1 t} \\
\Delta F_{t}= & c_{20}+\sum_{i=1}^{k} a_{2 i} \Delta S_{t-i}+\sum_{j=1}^{k} b_{i j} \Delta F_{t-j} \\
& +\gamma_{2} Z_{t-1}+\varepsilon_{2 t}
\end{aligned}
$$

$$
\begin{aligned}
\Delta S_{t}= & c_{10}+\sum_{i=1}^{k} a_{1 i} \Delta S_{t-i}+\sum_{j=1}^{k} b_{i j} \Delta F_{t-j} \\
& +\gamma_{1}\left(Z_{t-1} / \sqrt{t}\right)+\varepsilon_{1 t} \\
\Delta F_{t}= & c_{20}+\sum_{i=1}^{k} a_{2 i} \Delta S_{t-i}+\sum_{j=1}^{k} b_{i j} \Delta F_{t-j} \\
& +\gamma_{2}\left(Z_{t-1} / \sqrt{t}\right)+\varepsilon_{2 t}
\end{aligned}
$$

should read:

$$
\begin{aligned}
\Delta S_{t}= & c_{10}+\sum_{i=1}^{k} a_{1 i} \Delta S_{t-i}+\sum_{j=1}^{k} b_{1 j} \Delta F_{t-j} \\
& +\gamma_{1} Z_{t-1}+\varepsilon_{1 t} \\
\Delta F_{t}= & c_{20}+\sum_{i=1}^{k} a_{2 i} \Delta S_{t-i}+\sum_{j=1}^{k} b_{2 j} \Delta F_{t-j} \\
& +\gamma_{2} Z_{t-1}+\varepsilon_{2 t} \\
\Delta S_{t}= & c_{10}+\sum_{i=1}^{k} a_{1 i} \Delta S_{t-i}+\sum_{j=1}^{k} b_{1 j} \Delta F_{t-j} \\
& +\gamma_{1}\left(Z_{t-1} / \sqrt{t}\right)+\varepsilon_{1 t} \\
\Delta F_{t}= & c_{20}+\sum_{i=1}^{k} a_{2 i} \Delta S_{t-i}+\sum_{j=1}^{k} b_{2 j} \Delta F_{t-j} \\
& +\gamma_{2}\left(Z_{t-1} / \sqrt{t}\right)+\varepsilon_{2 t}
\end{aligned}
$$

